\title{
The anti-inflammatory effects of Yunnan Baiyao are involved in regulation of the phospholipase A2/arachidonic acid metabolites pathways in acute inflammation rat model
}

\author{
XIAOBIN REN $^{1}$, MINGZHU ZHANG ${ }^{1}$, LINGXIANG CHEN ${ }^{1}$, WANLI ZHANG ${ }^{1}$, \\ YU HUANG ${ }^{2}$, HUAZHEN LUO $^{3}$, LING LI $^{4}$ and HONGBING HE ${ }^{1}$ \\ ${ }^{1}$ Department of Periodontology, The Affiliated Stomatology Hospital of Kunming Medical University, Kunming, \\ Yunnan 650031; ${ }^{2}$ Department of Oral Medicine, The First Hospital of Qujing, Qujing, Yunnan 655000; \\ ${ }^{3}$ Department of Oral Medicine, The First Hospital of Kunming, Kunming, Yunnan 650011; ${ }^{4}$ Biomedical Engineering \\ Research Center, Kunming Medical University, Kunming, Yunnan 650500, P.R. China
}

Received August 29, 2016; Accepted May 25, 2017

DOI: $10.3892 / \mathrm{mmr} .2017 .7104$

\begin{abstract}
The traditional Chinese medicine Yunnan Baiyao (YNB) has been reported to possess anti-inflammatory properties, however its mechanism of action remains unclear. It was previously reported that YNB ameliorated depression of arachidonic acid (AA) levels in a rat model of collagen-induced arthritis. In the current study, the capacity of YNB to ameliorate inflammation was compared in carrageenan-induced and AA-induced acute inflammation of the rat paw with celecoxib and mizolastine, respectively $(n=24$ per group). The capacity of YNB to affect the phospholipase A2 (PLA2)/AA pathway (using reverse transcription-quantitative polymerase chain reaction) and release of inflammatory lipid mediators (by ELISA) were investigated. Celecoxib ameliorated carrageenan-induced paw edema, and mizolastine ameliorated AA-induced rat paw edema. YNB alleviated paw edema and inhibited inflammatory cell infiltration in the two models. YNB inhibited production of 5-LOX AA metabolite leukotriene B4 (LTB4), and suppressed expression of 5-LOX, cytosolic PLA2 (cPLA2), 5-LOX-activating protein, and LTB4 receptor mRNA in the AA-induced inflammation model $(\mathrm{P}<0.05)$. YNB Inhibited the production of the COX-2 AA
\end{abstract}

Correspondence to: Dr Hongbing He, Department of Periodontology, The Affiliated Stomatology Hospital of Kunming Medical University, 1088 Haiyuan Road, Kunming, Yunnan 650031, P.R. China

E-mail: 1320058043@qq.com

Abbreviations: 5-LOX, 5-lipoxygenase; AA, arachidonic acid; cPLA2, cytosolic PLA2; COX-2, cyclooxygenase 2; FLAP, 5-LOX-activating protein; GCs, glucocorticoids; LTB4, leukotriene B4; PGE2, prostaglandin E2; PLA2, phospholipase A2; YNB, Yunnan Baiyao

Key words: inflammation, Yunnan Baiyao, paw edema, cyclooxygenase, 5-lipooxygenase, traditional Chinese medicine metabolite prostaglandin E2 (PGE2) and suppressed expression of COX-2, cPLA2, PGE2 mRNA in the carrageenan-induced inflammation mode $(\mathrm{P}<0.05)$. Taken together, the data suggest that modulation of COX and LOX pathways in AA metabolism represent a novel anti-inflammatory mechanism of YNB.

\section{Introduction}

Pathological autoimmunity, which can cause conditions such as arthritis, can be treated with glucocorticoids (GCs) and nonsteroidal anti-inflammatory drugs, however their use is often limited by the adverse effects they can cause and novel anti-inflammatory therapies are required $(1,2)$. Traditional herbal medicine has been widely used for treating various inflammatory diseases in Eastern Asia $(3,4)$. Yunnan Baiyao (YNB) is a widely used traditional Chinese herbal remedy, and has since been used to treat various conditions, including bleeding (5), ulcers (6), infection (7), hemangiosarcoma (8) and inflammatory bowel disease (9).

Liu et al (10) reported that YNB could reduce ulcer size and accelerate healing in aphthous stomatitis. In a mouse model of colitis, Li et al (9) also established that the immunosuppressive activity of YNB involved highly selective cytotoxicity towards $\mathrm{B}$ and $\mathrm{T}$ lymphocytes, and inhibition of tumor necrosis factor $\alpha$ and interferon $\gamma$ expression in the colonic mucosa and plasma. In a previous study, it was identified that YNB could reduce symptoms of rheumatoid arthritis in a mouse model (11). YNB-induced amelioration of foot paw swelling and inflammation was identified to be associated with reduced plasma levels of prostaglandin (PG) E2 (PGE2) and interleukin 1 $\beta$, and plasma metabolomics analysis indicated that changes in circulating levels of arachidonic acid (AA) were recovered as a result of YNB administration (11).

The phospholipase A2 (PLA2)/AA pathway is a pivotal regulator of inflammatory responses. AA, liberated from the membrane phospholipids by PLA2, acts as a substrate for cyclooxygenase-2 (COX-2) and 5-lipoxygenase (5-LOX) to produce eicosanoids, including PGs and leukotrienes (LTs); important 
inflammatory mediators responsible for characteristic symptoms of inflammation. COX-2, is an inducible enzyme, which is mainly expressed under pathological conditions such as inflammation. Activation of the COX-2 pathway causes PG production. 5-LOX is a major isozyme component of the LOX pathway, which catalyzes biosynthesis of LTs from AA (12). Hence, inhibition of COXs and LOX can modulate AA metabolism and inhibit inflammation. Cytosolic PLA2 (cPLA2) serves an important role in liberation of AA from membrane phospholipids $(13,14)$. Once AA is released, it is metabolized by COXs and 5-LOX pathways (15). The AA pathway generates potent inflammatory mediators: PGs and LTs (16). COX2 catalyzes the two step conversion of AA to PGH2. This transformation begins with formation of the endoperoxide PGG2, which is metabolized into PGH2. LOX catalyzes the incorporation of molecular oxygen into AA to generate 5-hydroperoxyeicosatetraenoic acid that is dehydrated into LTA4. LTA4 is further metabolized to bioactive LTs to LTB4 or LTC4 (17). Production of LTs from endogenous AA requires 5-LOX-activating protein (FLAP), which may serve an essential role in AA transfer to 5-LOX (18). FLAP acts as a scaffold for 5-LOX at the nuclear envelope, where it facilitates access of AA to 5-LOX (19). Gene knockout or pharmacological interference with FLAP fully abolishes the generation of 5-LOX-derived products implying its crucial role in LT biosynthesis $(20,21)$.

As the PLA2/AA pathway is crucial to the pathogenesis of inflammatory diseases, it was hypothesized that the anti-inflammatory effects of YNB may be mediated via regulation of AA metabolism, particularly the COX and 5-LOX pathways. Therefore, the present study focused on the anti-inflammatory mechanisms of YNB, and compared them with those of a selective COX-2 inhibitor, celecoxib, and the histamine receptor antagonist, mizolastine, in two animal models of acute inflammation; carrageenan-induced and AA-induced rat paw edema, respectively $(22,23)$.

\section{Materials and methods}

Animals. A total of 192 Specific-pathogen-free male Sprague-Dawley rats, aged between 7 and 8 weeks-old (weight, $244.01 \pm 10.7 \mathrm{~g}$ ), were provided by the Kunming Medical University Animal Center (Kunming, China). The animals were housed under a constant $12 \mathrm{~h}$ light/dark cycle in a temperature- and humidity-controlled room, and given free access to solid food and tap water for the duration of the study. Animals were allowed 7 days to adapt to the environment prior to commencement of the experiments. All procedures and animal experiments were approved by the Animal Care and Use Committee of Kunming Medical University (Kunming, China). The rats were sacrificed by cervical dislocation subsequent to blood collection from the abdominal aorta and all efforts were made to minimize suffering.

\section{Rat paw edema model construction and treatment}

AA-induced edema. A total of 96 rats were randomly divided into four groups; the control group, model group, mizolastine group and YNB group. Mizolastine and YNB group animals received continuous intragastric administration of $0.3 \mathrm{mg} / \mathrm{kg}$ mizolastine (Xian-Janssen Pharmaceutical, Ltd., Beijing, China) or 50 mg/ml YNB (Yunnan Baiyao Group Co.,
Ltd., Kunming, China), respectively, for 7 days, as previously described (11). Model group and control group animals received the same volume of normal saline. A total of $2 \mathrm{~h}$ after intragastric administration on day 7, the model group, mizolastine and YNB group animals were subcutaneously injected with $0.1 \mathrm{ml} \mathrm{AA}(1 \mathrm{mg} / \mathrm{ml}$; Sigma-Aldrich; Merck Millipore, Darmstadt, Germany) to the left paw, and injection of $0.1 \mathrm{ml}$ carbonate buffer solution (AA vehicle) to the right paw, as previously described (23). The control group received no injection.

Carrageenan-induced edema. A total of 96 rats were randomly divided into four groups; the control group, model group, celecoxib group and YNB group. Celecoxib and YNB group animals received continuous intragastric administration of $10 \mathrm{mg} / \mathrm{kg}$ celecoxib (Pfizer, Inc., New York, NY, USA) or $50 \mathrm{mg} / \mathrm{ml}$ YNB, respectively, for 7 days, as previously described (24). Model group and control group animals received the same volume of normal saline. A total of $2 \mathrm{~h}$ after intragastric administration on day 7 , the model group, mizolastine and YNB group animals were subcutaneously injected with $0.15 \mathrm{ml}$ carrageenan $(150 \mu \mathrm{g}$ paw $^{-1}$; Sigma-Aldrich; Merck Millipore) to the left paw and $0.15 \mathrm{ml}$ saline to the right paw as a control. The control group received no injection (22).

Edema measurements. The volume (in $\mathrm{ml}$ ) of the hind paws of control and treated animals were measured with a plethysmometer (model 7150; Ugo Basile, S.R.L., Monvalle, Italy) at 1, 2, 3 and $4 \mathrm{~h}$ after stimulus injection. Results are presented as the difference in volume between the test paw and the control paw for each animal at the time shown. Edema $(\Delta \mathrm{V})$ was calculated as follows: $\Delta \mathrm{V}=\mathrm{Vt}-\mathrm{Vc}$, where $\mathrm{Vt}=\mathrm{is}$ the edema volume of the left paw; $\mathrm{Vc}=$ is the edema volume of the right paw. Edema inhibitory activity was calculated according to the following formula. Percentage inhibition $(\%)=\left(\Delta \mathrm{V}_{\text {model }}-\Delta \mathrm{V}_{\text {treated }}\right) / \Delta \mathrm{V}_{\text {model }} \mathrm{x} 100(25)$.

Histological analysis. Inflammation paws were collected and fixed in $70 \%$ ethanol for $24 \mathrm{~h}$ and then preserved in $10 \%$ formalin. Subsequently, the paws were dehydrated, blocked in paraffin and then sectioned with a microtome (5 $\mu \mathrm{m})(26)$. The sections were stained with haematoxylin and eosin for the evaluation of histopathological changes.

Reverse transcription-quantitative polymerase chain reaction $(R T-q P C R)$. Total RNA was isolated from edema soft tissue of inflamed and control paws using TRIzol reagent (Invitrogen; Thermo Fisher Scientific, Inc., Waltham, MA, USA) according to the manufacturer's protocol. RT reactions were performed using the PrimeScript ${ }^{\mathrm{TM}}$ 1st Strand cDNA synthesis kit (Takara Biotechnology Co., Ltd., Dalian, China). An equal amount of total RNA ( $2 \mu \mathrm{g})$ was used for cDNA synthesis according to the manufacturer's protocol. The reverse transcription reaction products were stored at $-20^{\circ} \mathrm{C}$. Primers for COX-2, PGE2, cPLA2, 5-LOX, LTB4r, FLAP and $\beta$-actin are described in Table I. The PCR primers were designed by Shanghai Sangon Biotechnology Co., Ltd. (Shanghai, China). PCR amplification was carried out using the gene-specific PCR primers, $2 \mu \mathrm{l} \mathrm{cDNA,} 12.5 \mu \mathrm{l} \mathrm{GoTaq}^{\circledR}$ Green Master Mix 2X 
Table I. Primer sequences for reverse transcription-quantitative polymerase chain reaction.

\begin{tabular}{|c|c|c|c|c|}
\hline \multirow[b]{2}{*}{ Pathway } & \multirow[b]{2}{*}{ Gene } & \multicolumn{2}{|c|}{ Sequence $\left(5^{\prime}-3^{\prime}\right)$} & \multirow[b]{2}{*}{ Product length (base pairs) } \\
\hline & & Forward primer & Reverse primer & \\
\hline \multirow[t]{5}{*}{ 5-LOX } & $5-\mathrm{LOX}$ & ATCTGGGTGCGTTCAAGTG & ATGTCCTCGGTGTTGTCCAT & 341 \\
\hline & LTB4r & TAGCTTTGTCGTGTGGAGCA & CAACGTCTTGTTCTTGGGTGT & 388 \\
\hline & cPLA2 & GCACATAATAGTGGAACACC & ACACAGTGCCATGCTGAACC & 530 \\
\hline & FLAP & GGCCATCGTCACCCTCATCA & GATGAGGCAGCGGGGAGA & 590 \\
\hline & $\beta$-actin & GCCATGTACGTAGCCATCCA & GAACCGCTCATTGCCGATAG & 190 \\
\hline \multirow[t]{4}{*}{$\operatorname{COX}$} & $\mathrm{COX}-2$ & TACCCGGACTGGATTCTACG & AGTCATCAGCCACAGGAGGA & 224 \\
\hline & PGE2 & GCATCTCATCTCTCCCAACG & ACTCGCAGCACACCATACAC & 228 \\
\hline & cPLA2 & GCACATAATAGTGGAACACC & ACACAGTGCCATGCTGAACC & 224 \\
\hline & $\beta$-actin & GCCATGTACGTAGCCATCCA & GAACCGCTCATTGCCGATAG & 323 \\
\hline
\end{tabular}

5-LOX, 5-lipoxygenase; $\mathrm{LTB}_{4}$ r, leukotriene B4 receptor; cPLA2, cytosolic phospholipase A2; FLAP, 5-LOX-activating protein; COX, cyclooxygenase; PGE2, prostaglandin E2.

Table II. Effects of YNB on carrageenan-induced rat paw edema.

\begin{tabular}{|c|c|c|c|c|}
\hline \multirow[b]{2}{*}{ Group } & \multicolumn{4}{|c|}{ Paw edema $(\Delta V)$ in $\mathrm{ml}(\%$ inhibition $)$} \\
\hline & $1 \mathrm{~h}$ & $2 \mathrm{~h}$ & $3 \mathrm{~h}$ & $4 \mathrm{~h}$ \\
\hline Control group & $0.02 \pm 0.01$ & $0.02 \pm 0.01$ & $0.02 \pm 0.01$ & $0.02 \pm 0.01$ \\
\hline Model group & $0.42 \pm 0.03^{\mathrm{a}}$ & $0.43 \pm 0.02^{\mathrm{a}}$ & $0.49 \pm 0.02^{\mathrm{a}}$ & $0.41 \pm 0.02^{\mathrm{a}}$ \\
\hline Celecoxib group & $0.29 \pm 0.04^{\mathrm{a}, \mathrm{b}}(31)$ & $0.22 \pm 0.03^{\mathrm{a}, \mathrm{b}}(50)$ & $0.22 \pm 0.03^{\mathrm{a}, \mathrm{b}}(55)$ & $0.20 \pm 0.03^{\mathrm{a}, \mathrm{b}}(51)$ \\
\hline YNB group & $0.37 \pm 0.02^{\mathrm{a}, \mathrm{b}}(12)$ & $0.31 \pm 0.02^{\mathrm{a}, \mathrm{b}}(28)$ & $0.29 \pm 0.02^{\mathrm{a}, \mathrm{b}}(41)$ & $0.29 \pm 0.02^{\mathrm{a}, \mathrm{b}}(29)$ \\
\hline
\end{tabular}

Paw edema was measured from 1 to $4 \mathrm{~h}$ following injection. Values are presented as the mean \pm standard error. The suppression rate is shown in parentheses. ${ }^{\mathrm{a}} \mathrm{P}<0.05$ vs. the control group; ${ }^{\mathrm{b}} \mathrm{P}<0.05$ vs. the model group. Each group at $1 \mathrm{~h}, \mathrm{n}=24$; each group at $2 \mathrm{~h}, \mathrm{n}=24$; each group at $3 \mathrm{~h}$, $\mathrm{n}=12$; each group at $4 \mathrm{~h}, \mathrm{n}=12$. YNB, Yunnan Baiyao.

Table III. Effects of YNB on arachidonic acid-induced rat paw edema.

\begin{tabular}{|c|c|c|c|c|}
\hline \multirow[b]{2}{*}{ Group } & \multicolumn{4}{|c|}{ Paw edema $(\Delta V)$ in $\mathrm{ml}$ (\% inhibition) } \\
\hline & $1 \mathrm{~h}$ & $2 \mathrm{~h}$ & $3 \mathrm{~h}$ & $4 \mathrm{~h}$ \\
\hline Control group & $0.05 \pm 0.02$ & $0.01 \pm 0.03$ & $0.02 \pm 0.04$ & $0.01 \pm 0.03$ \\
\hline Model group & $0.70 \pm 0.07^{\mathrm{a}}$ & $0.82 \pm 0.06^{\mathrm{a}}$ & $0.76 \pm 0.04^{\mathrm{a}}$ & $0.74 \pm 0.04^{\mathrm{a}}$ \\
\hline Mizolastine group & $0.55 \pm 0.05^{\mathrm{a}, \mathrm{b}}(21)$ & $0.67 \pm 0.04^{\mathrm{a}, \mathrm{b}}(18)$ & $0.65 \pm 0.05^{\mathrm{a}, \mathrm{b}}(14)$ & $0.63 \pm 0.03^{\mathrm{a}, \mathrm{b}}(15)$ \\
\hline YNB group & $0.57 \pm 0.05^{\mathrm{a}, \mathrm{b}}(19)$ & $0.71 \pm 0.05^{\mathrm{a}, \mathrm{b}}(13)$ & $0.65 \pm 0.06^{\mathrm{a}, \mathrm{b}}(14)$ & $0.64 \pm 0.04^{\mathrm{a}, \mathrm{b}}(14)$ \\
\hline
\end{tabular}

Paw edema was measured from 1 to $4 \mathrm{~h}$ after injection. Values are presented as the mean \pm standard error. The suppression rate is shown in parentheses. ${ }^{\mathrm{a}} \mathrm{P}<0.05$ vs. the control group; ${ }^{\mathrm{b}} \mathrm{P}<0.05$ vs. the model group. Each group at $1 \mathrm{~h}, \mathrm{n}=24$; each group at $2 \mathrm{~h}, \mathrm{n}=24$; each group at $3 \mathrm{~h}$, $\mathrm{n}=12$; each group at $4 \mathrm{~h}, \mathrm{n}=12$. YNB, Yunnan Baiyao.

(Promega Corporation, Madison, WI, USA) and $8.5 \mu 1 \mathrm{ddH}_{2} 0$. The reaction conditions for cPLA2, 5-LOX, LTB4r, FLAP and $\beta$-actin were 30 cycles, denaturation at $95^{\circ} \mathrm{C}$ for $30 \mathrm{sec}$, annealing at $60^{\circ} \mathrm{C}$ for $30 \mathrm{sec}$ and extension at $72^{\circ} \mathrm{C}$ for $10 \mathrm{~min}$. The reaction conditions for COX-2, PGE2, cPLA 2 and $\beta$-actin were 30 cycles, denaturation at $95^{\circ} \mathrm{C}$ for $30 \mathrm{sec}$, annealing at $59^{\circ} \mathrm{C}$ for $30 \mathrm{sec}$ and extension at $72^{\circ} \mathrm{C}$ for $10 \mathrm{~min}$. The PCR product was run on a $1.5 \%$ agarose gel and stained with ethidium bromide. Results are presented as ratios of target mRNA to $\beta$-actin mRNA. The band intensity was determined by Quantity One v4.6.2 software (Bio-Rad Laboratories, Inc., Hercules, CA, USA). 
B

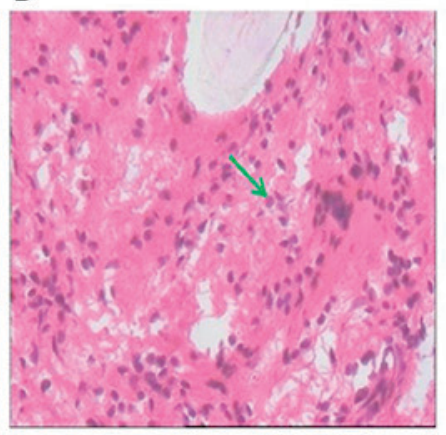

E

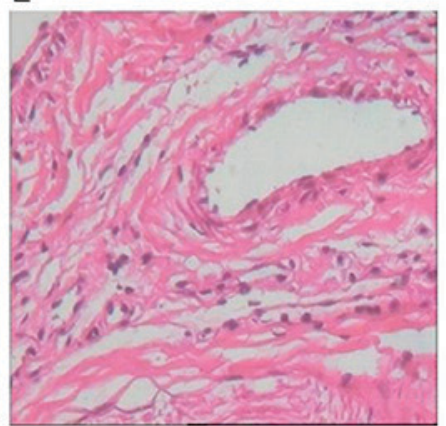

$\mathrm{H}$

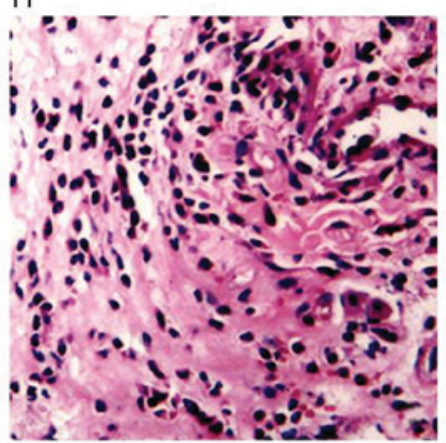

K

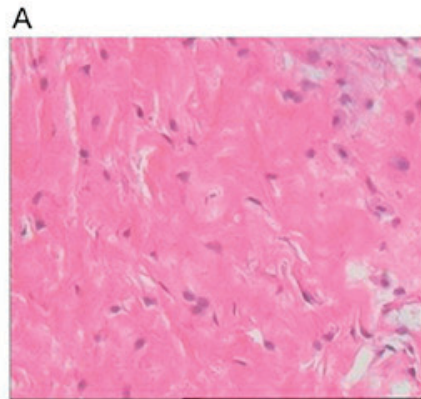

C

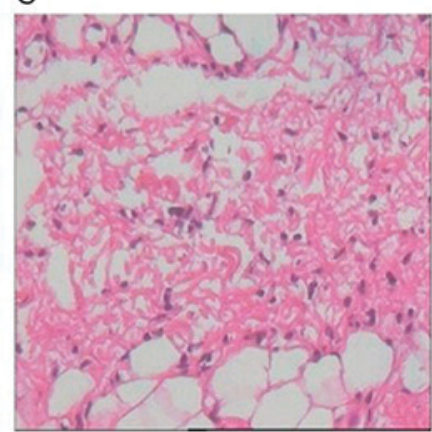

F

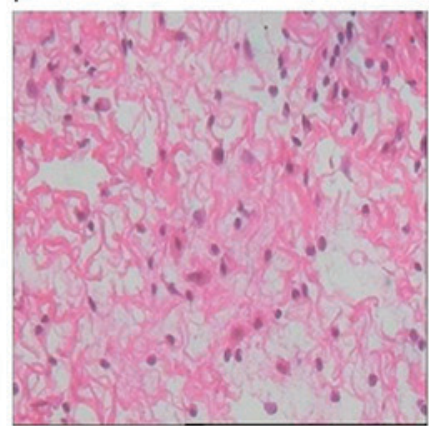

G

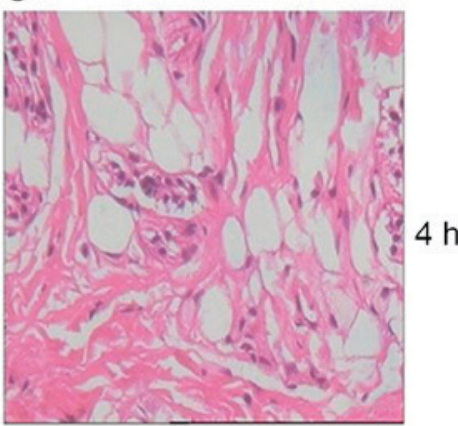

$4 \mathrm{~h}$
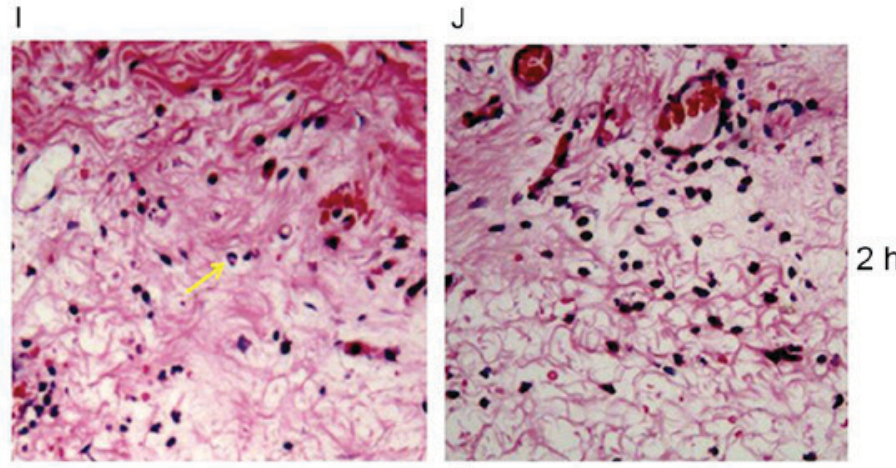

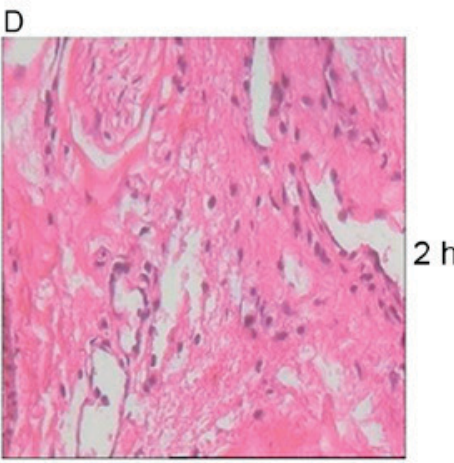

$2 \mathrm{~h}$
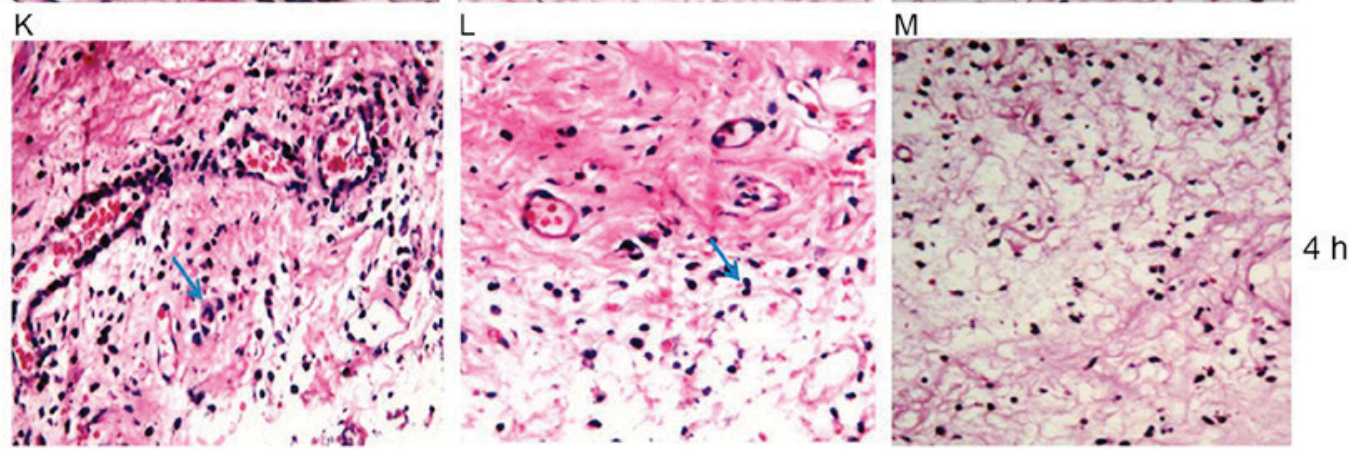

Figure 1. Paw inflammation in control, model and YNB, celecoxib and mizolastine rats. (A) H\&E stain of control paw from control group. H\&E staining of the inflamed paws was conducted in the carrageenan-induced inflammation model at (B-D) $2 \mathrm{~h}$ and (E-G) $4 \mathrm{~h}$. Inflamed paws from the (B and E) model group, (C and F) celecoxib group and (D and G) YNB group are presented. H\&E staining was additionally conducted in the arachidonic acid-induced inflammation model for (H-J) $2 \mathrm{~h}$ and (K-M) $4 \mathrm{~h}$. The inflamed paws from the (H and K) model group, (I and L) mizolastine group and (J and M) YNB group are presented. Inflammatory cell infiltration was observed in the model groups. Inflammation was alleviated in animals administered with YNB, celecoxib and mizolastine. Green arrows indicate leukomonocytes, blue arrows indicate eosnophils and yellow arrows indicate neutrophils. $\mathrm{n}=12$ in each group; magnification, $\mathrm{x} 400$. YNB, Yunnan Baiyao; H\&E, hematoxylin and eosin. 
ELISA. After rat paw volume was measured using a plethysmometer 1,2, 3 and $4 \mathrm{~h}$ after injection. A total of 12 rats were randomly taken from each group at 2 and $4 \mathrm{~h}$, anesthetized with $10 \%$ chloral hydrate, and sacrificed after blood was collected from the abdominal aorta. Rat blood was centrifuged for $10 \mathrm{~min}$ and the plasma was collected. ELISA kits (Sigma-Aldrich; Merck Millipore) were used to detect plasma levels of LTB4, cPLA2 and 5-LOX in the AA-induced inflammation model and PGE2, cPLA2 and COX-2 in the carrageenan-induced inflammation model, as instructed by the manufacturer.

Statistical analysis. The Experimental Data was analyzed using SPSS version 17.0 (SPSS, Inc., Chicago, IL, USA) All data were present as the mean \pm standard error and statistical analysis was performed using analysis of variance followed by Dunnett's test. $\mathrm{P}<0.05$ was considered to indicate a statistically significant difference.

\section{Results}

The acute anti-inflammatory effects of YNB in carrageenan-induced rat paw edema. The acute anti-inflammatory effects of celecoxib and YNB were investigated in the model of carrageenan-induced edema. In rats administered with carrageenan, significant paw edema was observed from $1 \mathrm{~h}$ after injection to $4 \mathrm{~h}$ after injection in the sub plantar region of the paw. In animals administered celecoxib paw edema was significantly reduced by $31 \%$ at $1 \mathrm{~h}$, and by $50-55 \%$ between 2 and $4 \mathrm{~h}$ after injection. In animals administered YNB paw edema was also significantly reduced by $12 \%$ at $1 \mathrm{~h}, 28 \%$ at $2 \mathrm{~h}, 41 \%$ at $3 \mathrm{~h}$ and $29 \%$ at $4 \mathrm{~h}$ (Table II), indicating that YNB has similar anti-inflammatory effects to celecoxib.

The acute anti-inflammatory effects of $Y N B$ on AA-induced rat paw edema. AA-induced rat paw edema was also used to evaluate anti-inflammatory activity. In rats administered with AA significant paw edema was observed from $1 \mathrm{~h}$ after injection to $4 \mathrm{~h}$ after injection in the sub plantar region of paw. In animals administered with mizolastine paw edema was significantly reduced by $21 \%$ at $1 \mathrm{~h}$, and by $14-18 \%$ between 2 and $4 \mathrm{~h}$ after injection $(\mathrm{P}<0.05$, Table III). In animals administered with YNB paw edema was also significantly reduced; by $19 \%$ at $1 \mathrm{~h}, 13 \%$ at $2 \mathrm{~h}, 14 \%$ at $3 \mathrm{~h}$ and $14 \%$ at $4 \mathrm{~h}(\mathrm{P}<0.05$, Table III), indicating that YNB has similar anti-inflammatory effects compared with mizolastine.

$Y N B$ inhibited inflammatory cell infiltration. In order to evaluate the protective effect of YNB on the histological changes induced in paw tissue during inflammation, the inflamed paw tissue was subjected to H\&E staining (Fig. 1). Histopathological changes were observed in the paws of rats administered carrageenan and AA. In the paw tissues of animals in the control group (Fig. 1A) a small number of inflammatory cells were observed in the subcutaneous tissue by light microscopy. In the paw tissues of animals in the model groups (Fig. 1B, E, $\mathrm{H}$ and $\mathrm{K}$ ) numerous inflammatory cells were observed in the subcutaneous tissue. In animals administered with YNB (Fig. 1D, G, J and M), celecoxib (Fig. 1I and L) or mizolastine (Fig. 1C and F) significantly reduced inflammatory cell

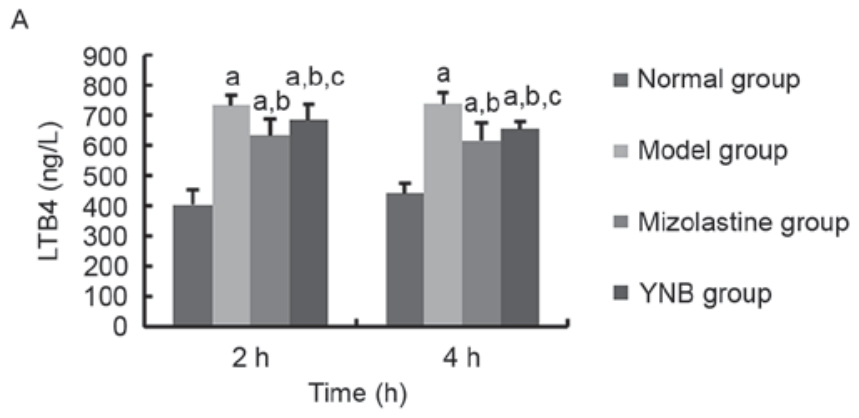

B
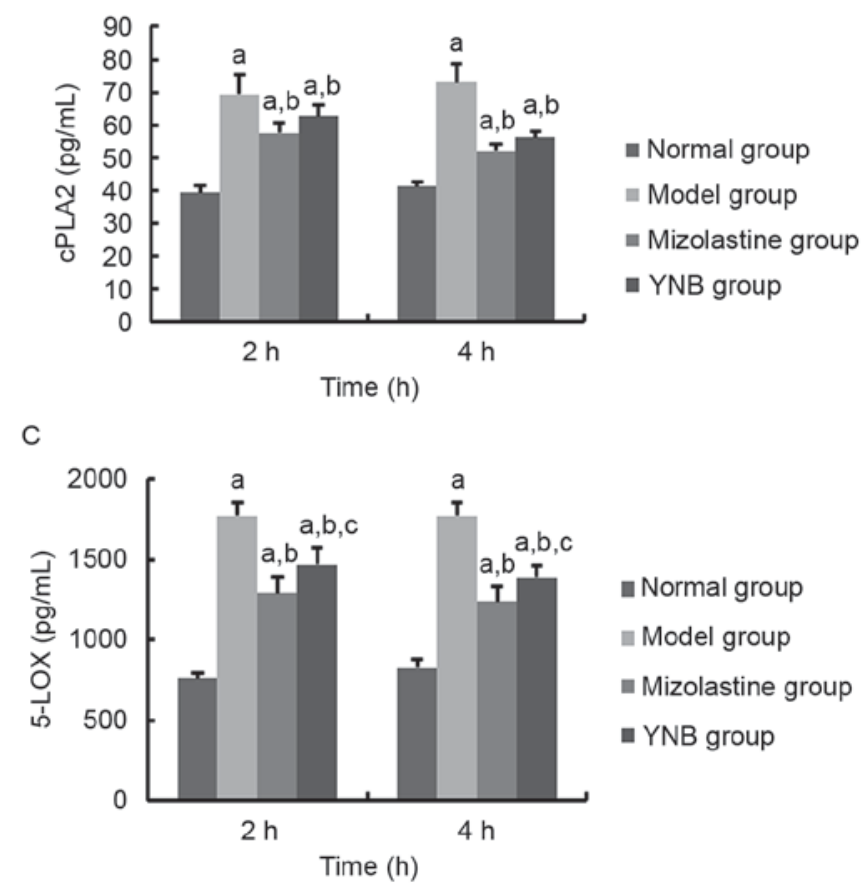

Figure 2. Basic serum biochemical parameters of control, model, YNB and mizolastine groups. Plasma levels of (A) LTB4, (B) cPLA2 and (C) 5-LOX in the arachidonic acid-induced inflammation model were assessed by ELISA 2 and 4 h subsequent to model establishment. Values are presented as the mean \pm standard error. ${ }^{\text {a }} \mathrm{P}<0.05$ vs. the control group; ${ }^{b} \mathrm{P}<0.05$ vs. the model group; ${ }^{\mathrm{c}} \mathrm{P}<0.05$ vs. the mizolastine or celecoxib groups. Each group, $\mathrm{n}=12$. YNB, Yunnan Baiyao; LTB4, leukotriene B4; cPLA2, cytosolic phospholipase A2; 5-LOX, 5-lipoxygenase.

infiltration was observed, indicating that YNB, celecoxib and mizolastine reduce inflammatory cell infiltration.

YNB inhibited the plasma levels of 5-LOX AA metabolite pathway key enzymes and inflammatory lipid mediators in the AA-induced inflammation model. In order to investigate the systemic cytokine response to AA-induced inflammation, plasma levels of LTB4, cPLA2 and 5-LOX were measured at 2 and $4 \mathrm{~h}$ by ELISA. Plasma levels of these proteins were all elevated in the AA-induced inflammation model (Fig. 2), both mizolastine and YNB resulted in significantly reduced levels of LTB4 (Fig. 2A; P<0.001), PLA $_{2}$ (Fig. 2B; P<0.001) and 5-LOX (Fig. 2C; $\mathrm{P}<0.001$ ), and levels were lower in the mizolastine group compared with the YNB group $(\mathrm{P}<0.05)$.

YNB suppressed expression of 5-LOX, cPLA2, FLAP and LTB4r mRNA in the AA-induced inflammation model. The capacity of YNB to suppress mRNA levels of key enzymes 
A

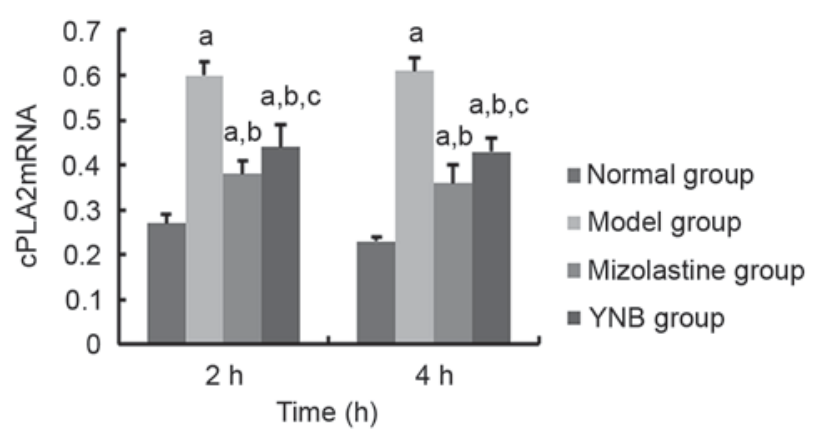

C

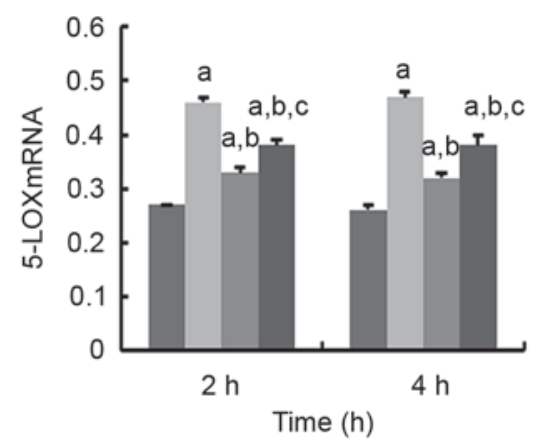

B

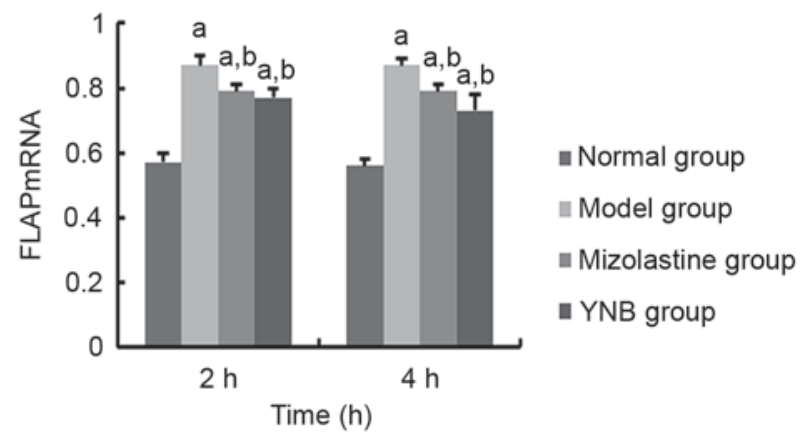

D

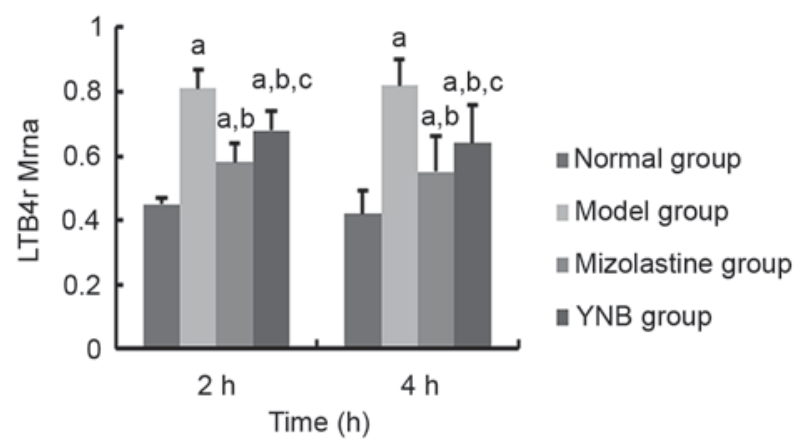

Figure 3. Expression of cPLA2 (A), FLAP (B), 5-LOX (C) and LTB4r (D) in arachidonic acid-induced edema model. mRNA levels were assessed by reverse transcription-quantitative polymerase chain reaction, 2 and $4 \mathrm{~h}$ following injection of arachidonic acid. Values are presented as the mean \pm standard error. ${ }^{\mathrm{a}} \mathrm{P}<0.05$ vs. the control group; ${ }^{\mathrm{b}} \mathrm{P}<0.05$ vs. the model group; ${ }^{\mathrm{c}} \mathrm{P}<0.05$ vs. the mizolastine group. $\mathrm{n}=12 \mathrm{per}$ group. cPLA2, cytosolic phospholipase $\mathrm{A} 2$; FLAP, 5-LOX-activating protein; 5-LOX, 5-lipoxygenase; LTB4r, leukotriene B4 receptor; YNB, Yunnan Baiyao.

of 5-LOX AA metabolite pathways was investigated. AA significantly increased levels of 5-LOX, cPLA2, FLAP and LTB4r mRNA in the model group (Fig. 3A-C; $\mathrm{P}<0.001$ ) compared with the control group. Model group and YNB significantly decreased levels of cPLA2 (Fig. 3A; P<0.001), FLAP (Fig. 3B; P<0.01), 5-LOX mRNA (Fig. 3C; $\mathrm{P}<0.001$ ) and LTB4r (Fig. 3D; $\mathrm{P}<0.01)$ compared with the model group. The suppressive effect of mizolastine was stronger than that of YNB on expression of cPLA2 (Fig. 3A; P<0.01), 5-LOX (Fig. 3C; $\mathrm{P}<0.001$ ) and LTB4r (Fig. 3D; P<0.05).

$Y N B$ inhibited the plasma levels of COX-2 AA metabolite pathway key enzymes and inflammatory lipid mediators in the carrageenan-induced inflammation model. To investigate the systemic cytokine response to carrageenan induced inflammation, plasma levels of PGE2, cPLA2 and COX-2 were measured at 2 and $4 \mathrm{~h}$ by ELISA. Plasma levels of these proteins were all elevated in the carrageenan-induced inflammation model (Fig. 4), both celecoxib and YNB significantly reduced levels of PGE2 (Fig. 4A; $\mathrm{P}<0.001$ ) in $4 \mathrm{~h}, \mathrm{cPLA}_{2}$ (Fig. 4B; $\mathrm{P}<0.001$ ) and COX-2 (Fig. 4C; $\mathrm{P}<0.001$ ) and levels were lower in the celecoxib group compared with the YNB group $(\mathrm{P}<0.001)$.

YNB suppressed expression of COX-2, cPLA2, PGE2 mRNA in the carrageenan-induced inflammation model. The capacity of YNB to suppress mRNA levels of key enzymes of the COX-2 AA metabolite pathway was investigated. Injection of carrageenan significantly increased plasma levels of COX-2, cPLA2 and PGE2 mRNA compared with the control group
(Fig. 5; $\mathrm{P}<0.001$ ). Administration of celecoxib significantly reduced levels of COX-2, cPLA2 in the $2 \mathrm{~h}$ group, and PGE2 mRNA compared with model group (Fig. 5; P<0.001). Administration of YNB also significantly reduced levels of COX-2 (Fig. 5A; P<0.001), PGE2 (Fig. 5; P<0.001) and cPLA2 (Fig. $5 \mathrm{C} ; \mathrm{P}<0.001$ ) in the $2 \mathrm{~h}$ group. The suppressive effect of celecoxib was stronger than that of YNB on expression of COX-2 $(\mathrm{P}<0.05)$ in the $2 \mathrm{~h}$ group, $\operatorname{cPLA} 2(\mathrm{P}<0.001)$ in $4 \mathrm{~h}$ group and $\mathrm{PGE} 2(\mathrm{P}<0.05)($ Fig. 5).

\section{Discussion}

Glucocorticoids (GCs) and nonsteroidal anti-inflammatory drugs can effectively reduce inflammation; however adverse effects limit their use. Therefore, efforts have been made to identify novel anti-inflammatory therapies. YNB a herbal medicine, has been used to treat various conditions, including bleeding, ulcers, infections, hemangiosarcoma and inflammatory bowel disease. In a previous study, the effect of YNB was investigated in a mouse model of collagen-induced arthritis, and it was identified that YNB significantly ameliorated the elevated plasma levels of AA (11). It was thus hypothesized that YNB may interfere with AA metabolic pathways. Thus, in the current study, two traditional models of acute inflammation were used, carrageen-induced paw edema and AA-induced paw edema, to evaluate the effect of YNB on AA metabolic pathways.

Carrageenan-induced paw edema is a well-established model for evaluating the biphasic events of acute inflammation. In the early phase, $0-2 \mathrm{~h}$ subsequent to carrageenan 


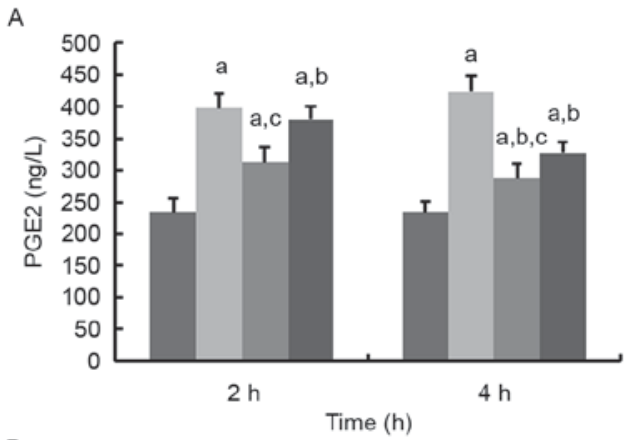

B
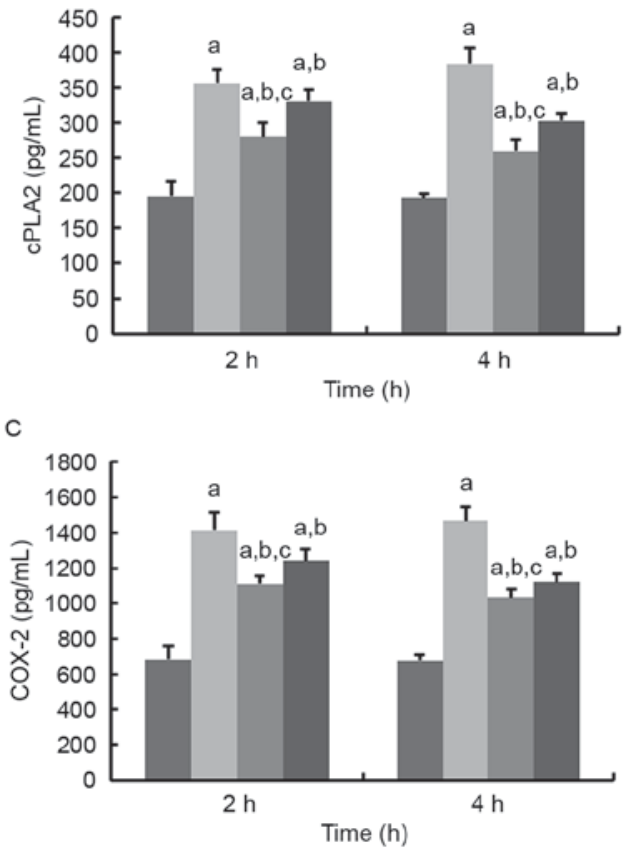

- Normal group =Model group - Celecoxib group - YNB group

- Normal group "Model group ॥ Celecoxib group $\because$ YNB group

- Normal group $=$ Model group - Celecoxib group $=$ YNB group

Figure 4. Basic serum biochemical parameters of control, model, YNB and celecoxib groups. Plasma levels of (A) PGE2, (B) cPLA2, (C) COX-2 in the carrageenan-induced inflammation model were assessed by ELISA 2 and $4 \mathrm{~h}$ subsequent to model establishment. Values are presented as the mean \pm standard error. ${ }^{\mathrm{P}} \mathrm{P}<0.05$ vs. the control group; ${ }^{\mathrm{b}} \mathrm{P}<0.05$ vs. the model group; ${ }^{\mathrm{P}} \mathrm{P}<0.05$ vs. the celecoxib group. $\mathrm{n}=12$ per group. $\mathrm{PGE} 2$, prostaglandin E2; cPLA2, cytosolic phospholipase A2; COX-2, cyclooxygenase 2; YNB, Yunnan Baiyao.

injection, histamine release induces vascular permeability. The second phase subsequent to injection of carrageenan is predominantly attributed to enhanced production of PGs. Inflammatory edema has been previously reported to peak $3 \mathrm{~h}$ following carrageenan injection (27), which was supported by the results of the current study; paw edema was observed in rats administered carrageenan injection after $1 \mathrm{~h}$, and edema peaked at $3 \mathrm{~h}$ and increased local inflammatory cell infiltration. The selective COX-2 inhibitor, celecoxib, ameliorated carrageenan-induced paw edema. AA-induced hind paw edema is another model of acute inflammation, and is ameliorated by the histamine receptor antagonist, mizolastine, however not by COX-inhibitors (28). This model is thus used to investigate the mechanisms of lipoxygenase inhibition. In the present study, paw edema was observed $1 \mathrm{~h}$ after AA injection, and peaked after $2 \mathrm{~h}$. Histological analysis indicated increased local inflammatory cell infiltration. AA is predominantly metabolized via the COX-2 and the 5-LOX pathways, leading to the formation of prostanoids and LTs, respectively.

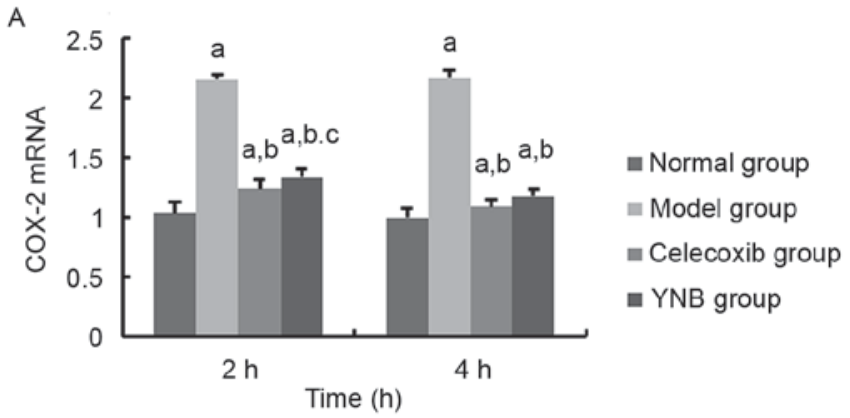

B

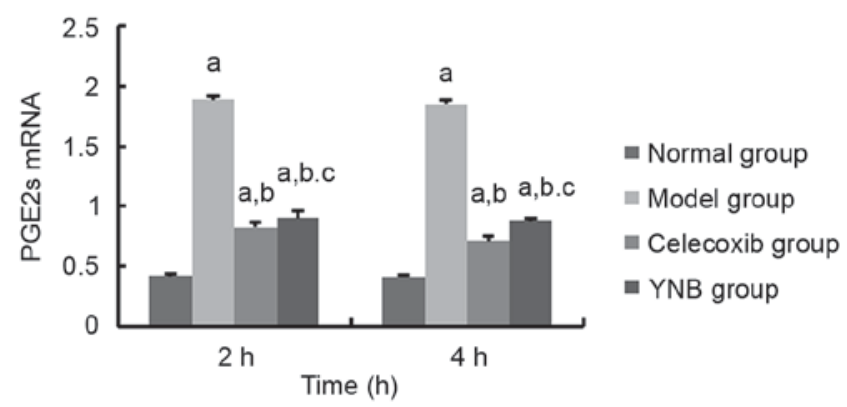

C

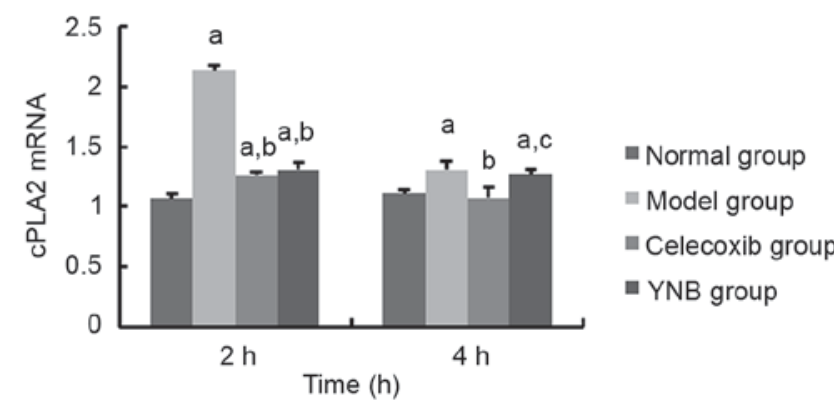

Figure 5. Expression of (A) COX-2, (B) PGE2, (C) cPLA2 in carrageenan-induced edema model. mRNA levels were assessed by reverse transcription-quantitative polymerase chain reaction, 2 and $4 \mathrm{~h}$ subsequent to injection of carrageenan. Values are presented as the mean \pm standard error. ${ }^{a} \mathrm{P}<0.05$ vs. the control group; ${ }^{\mathrm{b}} \mathrm{P}<0.05$ vs. the model group; ${ }^{\mathrm{C}} \mathrm{P}<0.05$ vs. the celecoxib group. $n=12$ per group. COX-2, cyclooxygenase 2; PGE2, prostaglandin E2; cPLA2, cytosolic phospholipase A2; YNB, Yunnan Baiyao.

The carrageen-induced paw edema and AA-induced paw edema activate the COX and 5-LOX pathways, respectively. Thus, these two models were used to investigate whether YNB can alleviate inflammation by inhibiting COX-2 and 5-LOX AA metabolites pathways.

cPLA2 induces AA release from cellular membranes. Blockade of PLA2 thus can reduce inflammation, and PLA2 inhibitors have become a pharmaceutical research focus (29). It was observed that carrageen and AA markedly elevated plasma levels of cPLA2. As predicted, YNB alleviated cPLA2 generation in the two models. Furthermore, YNB significantly reduced levels of cPLA2 mRNA in the paw. These data indicated that YNB could suppress expression of cPLA2 in the transcriptional level and explained why it enabled recovery of AA to normal levels in a previous study (11).

In the present study, it was identified that YNB alleviated paw edema and inhibited inflammatory cell infiltration in the two models. In order to investigate the mechanism of YNB action, the expression of inflammatory mediators was 
investigated in the two models. In rats administered with carrageenan, elevated circulating levels of $\mathrm{PGE}_{2}, \mathrm{cPLA}_{2}$ and $\mathrm{COX}-2$ were observed, and elevated levels of COX-2, cPLA2, PGE2 mRNA were identified in the paw. YNB inhibited the production of COX-2 AA metabolites, and suppressed expression of COX-2, cPLA2 and PGE2 mRNA although less effectively than that of celecoxib. In the present study, it was illustrated that carrageenan markedly activated the COX-2 pathway, while YNB ameliorated carrageenan-induced expression of PGE2 synthase, and reduced production of PGE2, which is consistent with the results of a previous study (11). In contrast, AA activated the 5-LOX pathway. In rats administered with AA we observed elevated circulating levels of LTB4, cPLA2 and 5-LOX, and elevated expression of cPLA2, FLAP, 5-LOX and LTB4r in the paw. YNB inhibited production of 5-LOX AA metabolites and suppressed the expression of 5-LOX, cPLA2, FLAP and LTB4r mRNA, although less effectively than that of mizolastine. COX-2 and 5-LOX are dual inhibitors that act on the two major AA metabolic pathways, and have been previously reported to possess superior anti-inflammatory activities (30-32). The data of the current study indicate that YNB can alleviate inflammation by inhibiting COX-2 and 5-LOX pathways. LTs serve a key role in the pathophysiology of inflammatory diseases and host defense reactions. LTB4, a potent chemotactic and chemokinetic mediator, can stimulate migration and activation of granulocytes to induce granulocyte adherence to vessel walls, degranulation, release of superoxide and lysosomal enzymes. Furthermore, LTB4 is associated with increased interleukin production and transcription (33). However, the effects of LTB4 are all mediated through binding to its receptor (LTB4r). The current study observed that YNB significantly ameliorated elevated plasma levels of LTB4 and LTB4r expression in the inflamed paw. LT synthesis requires FLAP, an $18 \mathrm{kDa}$ membrane-bound protein (34). 5-LOX and FLAP are both required for cellular LT synthesis (35). Thus, FLAP expression was analyzed in the inflamed paw. YNB markedly suppressed the expression of FLAP, suggesting that the anti-inflammatory effect of YNB may be partly caused by FLAP inhibition. As LT formation was not an absolute requirement for FLAP, and FLAP can also stimulate the utilization of exogenous AA by 5-LOX, FLAP can bind AA and other cis-unsaturated fatty acids facilitating the presentation of substrate for 5-LOX for optimal synthesis of LTA4. Therefore, the inhibitory effect of YNB on LTB4 may reflect inhibition of FLAP expression. Furthermore, LTB4 is a potent chemotactic factor for neutrophils, stimulates adhesion of leukocytes to vascular endothelia for extravasation into adjacent tissue, and induces neutrophil degranulation (36). These observations may explain why YNB reduced inflammatory cell infiltration in inflamed paw tissue.

YNB can be prepared in several ways (powder, capsule, aerosol and tincture) for local or oral application. Although the precise herbal formula of YNB is a trade secret, the product is now labeled to identify the major components of each $0.5 \mathrm{~g}$ serving (37). The 2011 manufacturer's label listed the following compounds; $200 \mathrm{mg}$ Tienchi ginseng root (Panax notoginseng), $85 \mathrm{mg}$ Ajuga forrestii Diels plant, $66.5 \mathrm{mg}$ Chinese yam root, $57.5 \mathrm{mg}$ Dioscoreae nipponica Makino root, $36 \mathrm{mg}$ Erodium stephanianum and Geranium wilfordii plant, $30 \mathrm{mg}$ Dioscoreae parvilora ting root and $25 \mathrm{mg}$ Inula cappa plant (5). Which of these components is responsible for the anti-inflammatory activities observed in the present study remains unclear.

In conclusion, YNB alleviated inflammation by inhibiting activation of key AA metabolism enzymes including cPLA2, COXs, PGE2 and 5-LOX, and release of AA pathway-generated inflammatory lipid mediators in two rat models of inflammation. The current study suggests that YNB may modulate cPLA2/AA pathways to reduce inflammation. These results provide a theoretical foundation for the clinical application of YNB in inflammatory diseases.

\section{Acknowledgements}

The present study was supported by the National Natural Science Foundation of China (grant no. 81060086) and the Nature Science Foundation of Yunnan Province (grant no. 2012FB008).

\section{References}

1. Woodworth TG and den Broeder AA: Treating to target in established rheumatoid arthritis: Challenges and opportunities in an era of novel targeted therapies and biosimilars. Best Pract Res Clin Rheumatol 29: 543-549, 2015.

2. Bijlsma JW, Jacobs JW and Buttgereit F: Glucocorticoids in the treatment of rheumatoid arthritis. Clin Exp Rheumatol 33 (4 Suppl 92): S34-S36, 2015.

3. Xue $\mathrm{T}$ and Roy R: Studying traditional Chinese medicine. Science 300: 740-741, 2003.

4. Chrubasik JE, Roufogalis BD and Chrubasik S: Evidence of effectiveness of herbal antiinflammatory drugs in the treatment of painful osteoarthritis and chronic low back pain. Phytother Res 21: 675-683, 2007.

5. Radmark O, Werz O, Steinhilber D and Samuelsson B: 5-Lipoxygenase, a key enzyme for leukotriene biosynthesis in health and disease. Biochim Biophys Acta 1851: 331-339, 2015.

6. Ladas EJ, Karlik JB, Rooney D, Taromina K, Ndao DH, Granowetter L and Kelly KM: Topical Yunnan Baiyao administration as an adjunctive therapy for bleeding complications in adolescents with advanced cancer. Support Care Cancer 20: 3379-3383, 2012.

7. Yang B, Xu ZQ, Zhang H, Xu FY, Shi XY, Zou Z, Ling CQ and Tang L: The efficacy of Yunnan Baiyao on haemostasis and antiulcer: A systematic review and meta-analysis of randomized controlled trials. Int J Clin Exp Med 7: 461-482, 2014.

8. Wirth KA, Kow K, Salute ME, Bacon NJ and Milner RJ: In vitro effects of Yunnan Baiyao on canine hemangiosarcoma cell lines. Vet Comp Oncol 14: 281-294, 2016.

9. Li R, Alex P, Ye M, Zhang T, Liu L and Li X: An old herbal medicine with a potentially new therapeutic application in inflammatory bowel disease. Int J Clin Exp Med 4: 309-319, 2011.

10. Liu X, Guan X, Chen R, Hua H, Liu Y and Yan Z: Repurposing of Yunnan Baiyao as an alternative therapy for minor recurrent aphthous stomatitis. Evid Based Complement Alternat Med 2012: $284620,2012$.

11. He H, Ren X, Wang X, Shi X, Wang X, Ding Z, Gao P and Xu G: Therapeutic effect of Yunnan Baiyao on rheumatoid arthritis was partially due to regulating arachidonic acid metabolism in osteoblasts. J Pharm Biomed Anal 59: 130-137, 2012.

12. Charlier $\mathrm{C}$ and Michaux C: Dual inhibition of cyclooxygenase-2 $(\mathrm{COX}-2)$ and 5-lipoxygenase (5-LOX) as a new strategy to provide safer non-steroidal anti-inflammatory drugs. Eur J Med Chem 38: 645-659, 2003.

13. Murakami M, Taketomi Y, Miki Y, Sato H, Hirabayashi T and Yamamoto K: Recent progress in phospholipase $\mathrm{A}_{2}$ research: From cells to animals to humans. Prog Lipid Res 50: 152-192, 2011.

14. Cao J, Burke JE and Dennis EA: Using hydrogen/deuterium exchange mass spectrometry to define the specific interactions of the phospholipase A2 superfamily with lipid substrates, inhibitors, and membranes. J Biol Chem 288: 1806-1813, 2013. 
15. Magrioti V and Kokotos G: Phospholipase A2 inhibitors for the treatment of inflammatory diseases: A patent review (2010-present). Expert Opin Ther Pat 23: 333-344, 2013.

16. Mendes RT, Stanczyk CP, Sordi R, Otuki MF, dos Santos FA and Fernandes D: Selective inhibition of cyclooxygenase-2: Risks and benefits. Rev Bras Reumatol 52: 767-782, 2012.

17. Alexander SP, Benson HE, Faccenda E, Pawson AJ, Sharman JL, Spedding M, Peters JA and Harmar AJ; CGTP Collaborators: The concise guide to PHARMACOLOGY 2013/14: Enzymes. Br J Pharmacol 170: 1797-1867, 2013

18. Ferguson AD, McKeever BM, Xu S, Wisniewski D, Miller DK, Yamin TT, Spencer RH, Chu L, Ujjainwalla F, Cunningham BR, et al: Crystal structure of inhibitor-bound human 5-lipoxygenase-activating protein. Science 317: 510-512, 2007.

19. Bair AM, Turman MV, Vaine CA, Panettieri RA Jr and Soberman RJ: The nuclear membrane leukotriene synthetic complex is a signal integrator and transducer. Mol Biol Cell 23: 4456-4464, 2012

20. Byrum RS, Goulet JL, Griffiths RJ and Koller BH: Role of the 5-lipoxygenase-activating protein (FLAP) in murine acute inflammatory responses. J Exp Med 185: 1065-1075, 1997.

21. Evans JF, Ferguson AD, Mosley RT and Hutchinson JH: What's all the FLAP about? 5-lipoxygenase-activating protein inhibitors for inflammatory diseases. Trends Pharmacol Sci 29: 72-78, 2008.

22. Francischi JN, Chaves CT, Moura AC, Lima AS, Rocha OA, Ferreira-Alves DL and Bakhle YS: Selective inhibitors of cyclo-oxygenase-2 (COX-2) induce hypoalgesia in a rat paw model of inflammation. Br J Pharmacol 137: 837-844, 2002.

23. Pichat P, Angel I and Arbilla S: Anti-inflammatory properties of mizolastine after oral administration on arachidonic acid-induced cutaneous reaction in the rat. Arzneimittelforschung 48: 173-178, 1998.

24. Pinheiro RM and Calixto JB: Effect of the selective COX-2 inhibitors, celecoxib and rofecoxib in rat acute models of inflammation. Inflamm Res 51: 603-610, 2002.

25. Fabri RL, Garcia RA, Florêncio JR, de Castro Campos Pinto N, de Oliveira LG, Aguiar JA, Ribeiro A and Scio E: Anti-inflammatory and antioxidative effects of the methanolic extract of the aerial parts of Mitracarpus frigidus in established animal models. J Pharm Pharmacol 66: 722-732, 2014.

26. da Silva JM, Conegundes JL, Mendes Rde F, Pinto Nde C, Gualberto AC, Ribeiro A, Gameiro J, de Aguiar JA, Castañon MC and Scio E: Topical application of the hexane fraction of Lacistema pubescens reduces skin inflammation and cytokine production in animal model. J Pharm Pharmacol 67: 1613-1622, 2015.
27. Winter CA, Risley EA and Nuss GW: Carrageenin-induced edema in hind paw of the rat as an assay for antiiflammatory drugs. Proc Soc Exp Biol Med 111: 544-547, 1962.

28. DiMartino MJ, Campbell GK Jr, Wolff CE and Hanna N: The pharmacology of arachidonic acid-induced rat paw edema. Agents Actions 21: 303-305, 1987.

29. Miyaura C, Inada M, Matsumoto C, Ohshiba T, Uozumi N, Shimizu T and Ito A: An essential role of cytosolic phospholipase A2alpha in prostaglandin E2-mediated bone resorption associated with inflammation. J Exp Med 197: 1303-1310, 2003.

30. Julémont F, Dogné JM, Pirotte B and de Leval X: Recent development in the field of dual COX/5-LOX inhibitors. Mini Rev Med Chem 4: 633-638, 2004

31. Cianchi F, Cortesini C, Magnelli L, Fanti E, Papucci L, Schiavone N, Messerini L, Vannacci A, Capaccioli S, Perna F, et al: Inhibition of 5-lipoxygenase by MK886 augments the antitumor activity of celecoxib in human colon cancer cells. Mol Cancer Ther 5: 2716-2726, 2006

32. Ranjbar MM, Assadolahi V, Yazdani M, Nikaein D and Rashidieh B: Virtual Dual inhibition of COX-2/5-LOX enzymes based on binding properties of alpha-amyrins, the anti-inflammatory compound as a promising anti-cancer drug. EXCLI J 15: $238-245,2016$.

33. Fitzpatrick F, Haeggström J, Granström E and Samuelsson B: Metabolism of leukotriene A4 by an enzyme in blood plasma: A possible leukotactic mechanism. Proc Natl Acad Sci USA 80: 5425-5429, 1983.

34. Gravel J, Falgueyret JP, Yergey J, Trimble L and Riendeau D: Identification of 5-keto-(7E,9E,11Z,14Z)-eicosatetraenoic acid as a novel nonenzymatic rearrangement product of leukotriene A4. Arch Biochem Biophys 306: 469-475, 1993.

35. Reid GK, Kargman S, Vickers PJ, Mancini JA, Léveillé C, Ethier D, Miller DK, Gillard JW, Dixon RA and Evans JF: Correlation between expression of 5-lipoxygenase-activating protein, 5-lipoxygenase, and cellular leukotriene synthesis. J Biol Chem 265: 19818-19823, 1990.

36. Werz O: 5-lipoxygenase: Cellular biology and molecular pharmacology. Curr Drug Targets Inflamm Allergy 1: 23-44, 2002.

37. Wynn SG: Regulation and quality control in veterinary herbal medicine. In: Veterinary Herbal Medicine. Wynn SG and Fougere BJ (eds). Mosby Elsevier, St Louis, pp99-119, 2007. 